\title{
Pavlovian conditioning and extinction of context cues and punctate CSs in rats with ibotenate lesions of the hippocampus
}

\author{
STEPHEN C. BENOIT, T. L. DAVIDSON, and K.-H. CHAN \\ Purdue University, West Lafayette, Indiana \\ and \\ TIM TRIGILIO and LEONARD E. JARRARD \\ Washington \& Lee University, Lexington, Virginia
}

\begin{abstract}
The degree to which conditioned stimuli (CSs) control responding is often influenced when contextual cues are also associated with the unconditioned stimulus (US). In Experiment 1, selective ibotenate (IBO) lesions of the hippocampus impaired the ability of rats to inhibit responding to contextual cues made excitatory by presentation of unsignaled USs during simple Pavlovian CS-US training. Hippocampal damage had little effect on responding if few or no unsignaled USs had been delivered in the context. In Experiment 2, there was no evidence of increased responding to contextual cues when IBO lesions were given after excitatory training. However, lesioned rats showed greater resistance to CS extinction than did controls. An interpretation that encompasses these and other findings is that selective lesions of the hippocampus impair the formation of at least some types of inhibitory associations.
\end{abstract}

Impaired responding to a target conditioned stimulus (CS) is seen when extra unsignaled presentations of the unconditioned stimulus (US) occur during the intervals between CS-US training trials (Rescorla, 1968). The Rescorla-Wagner model explains this effect by proposing that such unsignaled US presentations result in the formation of an association between context stimuli and the US. This enables the context to compete successfully with the target CS for associative strength (Rescorla \& Wagner, 1972). One consequence of this mode of presenting unsignaled USs can be a reduction in the capacity of concurrent CS-US pairings to promote the performance of CRs (see, e.g., Rescorla, 1968). Apparently, the formation of a context-US association reduces the strength (e.g., Rescorla \& Wagner, 1972) or the effectiveness (e.g., Gibbon \& Balsam, 1980; Miller \& Matzel, 1988) of the CS-US association.

In a number of studies, researchers have examined the effects of removing the hippocampus, a medial temporal lobe structure often implicated in learning and memory processes, on the ability of rats to form either simple

We thank Curtis Chong for assistance in the collection of data and Javier Morell for his helpful comments during discussions of this work. Support for this research was provided by grants from the National Institutes of Health and the National Science Foundation, and, in part, by a National Science Foundation Pre-Doctoral Fellowship. Portions of these results were presented at the 66th and 67th annual meeting of the Society for Neuroscience. Correspondence concerning this article should be addressed to S. C. Benoit, Department of Psychological Sciences, Purdue University, West Lafayette, IN 47907 (e-mail: scbenoit@, psych.purdue.edur).
CS-US or context-US associations (e.g., Eichenbaum, Otto, \& Cohen, 1992; Honey \& Good, 1993; Rawlins, 1985; Seldon, Everitt, Jarrard, \& Robbins, 1991). Little is known, however, about how learning one type of association (e.g., context-US ) influences conditioned responding based on the other type of association (e.g., CS-US) for rats without a hippocampus. If, as some data suggest, hippocampally lesioned rats form abnormally strong contextUS associations (e.g., Winocur, Rawlins, \& Gray, 1987), context cues should evoke more conditioned responding and a concurrently trained CS-US relationship should evoke less conditioned responding in lesioned rats than in controls. In contrast, if lesions of the hippocampus impair learning about contextual stimuli (e.g., Honey \& Good, 1993; Philips \& LeDoux, 1992; Wilson, Brooks, \& Bouton, 1995), context stimuli would tend to evoke less conditioned responding and would be less able to interfere with response evocation by punctate CSs in rats without a hippocampus than in controls.

In Experiment 1, rats with selective ibotenate (IBO) lesions of the hippocampus were compared with controls in terms of their sensitivity to the effects of unsignaled USs on the behavioral consequences of simple Pavlovian CSUS training. Experiment 2 was intended to assess whether removal of the hippocampus influenced the acquisition or retention of this type of sensitivity.

\section{EXPERIMENT 1}

All rats were given simple Pavlovian excitatory conditioning in which a brief presentation of a tone CS was followed by presentation of a food US. One CS-US pair- 
ing was presented during each training session. In addition, some rats also received unsignaled presentations of the US (i.e., the US was delivered in the absence of the CS). Groups of rats differed with respect to the number of unsignaled USs that they received during Pavlovian CSUS excitatory conditioning. One group received zero unsignaled USs, another group received one unsignaled US every three training sessions, a third group received one unsignaled US each training session, and the fourth group received three unsignaled USs during each training session. One half of the rats in each group received IBO lesions of the hippocampus prior to training. Experiment 1 was an attempt to directly assess the effects of unsignaled US presentation on the context-US association by measuring conditioned activity during a pre-CS (i.e., responding in the presence of contextual cues alone; see Philips \& LeDoux, 1992) as well as responding during the CS for each group. Finally, the amount of conditioning to the CS was also assessed in a novel context (i.e., a context that should have little or no association with the US). If unsignaled US presentations reduce the effectiveness of CS-US presentations in hippocampally lesioned rats relative to controls, it might be expected that lesioned rats would exhibit reduced rates of CS conditioned responding relative to controls when tested in a novel context.

\section{Method}

\section{Subjects}

The subjects were 32 naive, male Sprague-Dawley albino rats (Harlan Sprague-Dawley, Indianapolis). Upon arrival, the rats were approximately 90 days old and weighed $275-300 \mathrm{~g}$. They were housed individually in stainless steel cages and were maintained on a 12:12-h light:dark cycle with lights on at $0700 \mathrm{~h}$. Rats were fed Purina rat chow, and water was available ad lib in the home cage throughout the experiment. Prior to the beginning of the experiment, the rats were gradually food deprived to $85 \%$ of free-feeding body weight. Sixteen animals received IBO lesions of the hippocampus, 8 were sham operated controls, and 8 were unoperated controls.

\begin{abstract}
Apparatus
All conditioning and testing procedures were conducted in four identical conditioning chambers, constructed of aluminum end walls and clear Plexiglas sides, measuring $21.6 \times 21.6 \times 27.9 \mathrm{~cm}$ (animals were run in training and test session in squads of 4 animals). The floor of each chamber consisted of stainless steel bars, $.48 \mathrm{~cm}$ in diameter, spaced $1.9 \mathrm{~cm}$ apart. A food cup was located in one end wall of each chamber. Each chamber was seated within a larger, sound-attenuating enclosure. The tone CS was produced by a Radio Shack Piezo Alerting Buzzer (No. 273-068) located outside of the chamber by the end wall with the food cup. These boxes served as training and test contexts. During training, the chambers were darkened and ambient noise was minimal. During novel context testing, the chambers were illuminated with exterior light, noise was added to the experimental room, and the grid floor was completely covered with fitted, black plastic panels.
\end{abstract}

\section{Data Collection}

Changes in behavioral activity were monitored by a computercontrolled infrared monitoring system. Sixteen electronic photobeams (ENV- 256C Infra-red Photobeam Controller and DIG-712 Photobeam Inputs, Med Associates) lined each cage from sidewall to sidewall approximately $2 \mathrm{~cm}$ above the grid floor. These beams were controlled, and the data analyzed, by software developed in the lab for measurement of generalized behavior. Approximately 30 times per second during the pre-CS and CS periods, the computer recorded binary values for photobeam interruptions in each box. Activity scores were determined by counting the number of times a change was recorded in photobeam interruptions (i.e., a behavioral activity count was recorded each time two temporally adjacent values were unequal, indicating movement within the box). Greater activity scores, then, indicated greater movement within the conditioning chamber. The computer and interface operating the photobeams were located in an adjoining room.

\section{Behavioral Procedure}

Training. Prior to training, 16 rats were randomly selected to receive IBO lesions of the hippocampus. Training began approximately 14 days after surgery was completed, and after the body weights of all rats were reduced to $85 \%$ of ad-lib feeding levels. All rats received 15 daily 50 -min training sessions in the training context. No events were programmed to occur during the first $20 \mathrm{~min}$ of each training session. During the final $30 \mathrm{~min}$ of each session, all rats received one 10 -sec tone CS immediately followed by a US (two 45-mg food pellets; Noyes, Lancaster, $\mathrm{NH}$ ). Behavioral activity, described above, was measured during each 10 -sec tone $\mathrm{CS}$ as well as the $10 \mathrm{sec}$ immediately preceding tone CS presentation.

In addition to the single tone CS-US pairing per session, three groups of rats also received unsignaled US presentations. Groups differed with respect to the number of unsignaled USs that were delivered in addition to the single tone CS-US pairing per session. Table 1 shows the design for Experiment 1. Groups are labeled in Table 1 with respect to the total number of unsignaled USs that were delivered during training. Group $0-$ US received 0 unsignaled USs during the course of training. Group 5-US received 1 unsignaled US delivery every three training sessions, for a total of 5 unsignaled USs during the course of training. Finally, Group 15-US received 1 unsignaled US presentation during each of the 15 training sessions, and Group 45-US received 3 unsignaled USs per training session. Within each group (0-US, 5-US, 15-US, and 45-US), one half of the animals had received IBO lesions of the hippocampus $(n=4)$. Unsignaled US presentation times were programmed to occur at intervals of no less than 2 min from other programmed events.

Novel context test. As described by Bouton (e.g., 1994), excitation to a punctate CS should readily transfer across different contexts. Thus, one way to assess conditioning to the tone CS is to test conditioned responding to that $\mathrm{CS}$ in a novel context. The day after training was completed, all rats received one preexposure session in a novel context to attenuate unconditioned behavioral activity. Following this preexposure session, all animals received three extinction test sessions (i.e., one tone CS but no US was delivered in the new context during each session). The contextual change was achieved by illuminating the training chambers, adding ambient noise, and placing black, plastic inserts across the grid floor. During these test sessions, all animals received one tone CS followed by no US. In addition, no group received any unsignaled presentations of the US. Conditioned behavioral activity was recorded during the 10 -sec tone $\mathrm{CS}$ as well as $10 \mathrm{sec}$ immediately preceding tone presentation.

\section{Surgical and Anatomical Procedures}

The procedures used for IBO lesioning of the hippocampus are described in detail elsewhere (Jarrard, 1989). Briefly, the animals were first anesthetized with chloral hydrate $(42 \mathrm{mg} / \mathrm{ml})$ and sodium pentobarbital $(9.7 \mathrm{mg} / \mathrm{ml})$ at a dose of $3 \mathrm{mg} / \mathrm{kg}$. Rats $(n=8)$ that received IBO lesions of the hippocampus were placed in a Kopf stereotaxic apparatus, an incision was made in the scalp, and the bone overlying the hippocampus was removed. Injections of IBO (Biosearch Technologies, San Rafael, CA) were made with a 5- $\mu 1$ Hamilton syringe mounted on the stereotaxic frame and held with 
Table 1

Experiment 1 Design

\begin{tabular}{ccccc}
\hline Group & $N$ & \multicolumn{1}{c}{ CS-US Training } & $\begin{array}{c}\text { Novel Context } \\
\text { US Exposure }\end{array}$ & $\begin{array}{c}\text { Training Context } \\
\text { CS Testing }\end{array}$ \\
\hline 0-US & 8 & 0 Unsignaled USs per session & US & CS- \\
5-US & 8 & 1 Unsignaled US per 3 sessions & US & CS- \\
15-US & 8 & 1 Unsignaled US per session & US & CS- \\
45-US & 8 & 3 Unsignaled USs per session & US & CS- \\
\hline
\end{tabular}

Note- One half of the rats in each group $(n=4)$ received ibotenate lesions of the hippocampus prior to training. Remaining rats were sham operated or unoperated controls. During training, all rats received one CS-US pairing per session. Training and test session length $=50 \mathrm{~min}$. CS $=10-\mathrm{sec}$ tone. $\mathrm{US}=$ two $45-\mathrm{mg}$ Noyes food pellets.

a Kopf microinjector (Model 5000). A glass micropipette was glued to the tip of the syringe needle in order to minimize damage to overlying cortical tissue. IBO was dissolved in phosphate-buffered saline $(\mathrm{pH} \mathrm{7.4)}$ at a concentration of $10 \mathrm{mg} / \mathrm{ml}$. Injections of $.10 \mu \mathrm{l}(.05$ at some sites) were made over approximately $1 \mathrm{~min}$ at each of 28 sites (see Jarrard, 1989, for coordinates). The pipette was left in place an additional $30 \mathrm{sec}$ at each site to prevent spread up the pipette track. The rats that received control operations were anesthetized as previously described, placed in the stereotaxic instrument, and had the overlying bone removed. A glass pipette was lowered through the cortex at the same sites as in rats receiving IBO lesions of the hippocampus, but no IBO was delivered.

After completion of the experiment, all lesioned animals were deeply anesthetized and perfused with physiological saline and formalin. The brains were removed from the skull, embedded in egg yolk, and cut in coronal (some horizontal) sections on a cryostat into $40-\mu \mathrm{m}$ sections. A cresyl violet stain was used to determine cell loss due to the lesions.

\section{Results \\ Training \\ Figure 1 shows mean activity score during the pre-CS period for the final three-trial block of acquisition. Higher scores indicate greater amounts of behavioral activity.}

Open bars represent activity scores for the animals with lesions of the hippocampus, while closed bars show activity scores for the controls. Note that the control animals exhibited approximately the same amount of activity regardless of the number of unsignaled USs (Groups 0-, 5-, 15-, and 45-US). However, the animals with lesions of the hippocampus exhibited greater pre-CS behavioral activity in Groups 15- and 45-US.

An alpha level of .05 was used throughout both experiments to assess significance. Newman-Keuls post hoc tests were conducted with an alpha level of .05. A mixed $2 \times 4 \times 2 \times 2$ analysis of variance (ANOVA) with lesion (hippocampal lesion vs. control), group (0-, 5-, 15 , and 45-US), training blocks (1-5), and period (pre-CS vs. CS) as factors was conducted on the activity scores recorded during acquisition. The ANOVA yielded a significant main effect of period $[F(1,24)=53.24, p<.01]$ as well as lesion $\times$ period $[F(1,24)=13.39, p<.01]$ and lesion $\times$ group $\times$ period $[F(3,24)=5.03, p<.01]$ interactions. Subsequent ANOVAs conducted on Groups 0 - and 5-US as well as 15 - and 45-US revealed no interactions between these groups. That is, Groups 0 - and 5-US were not

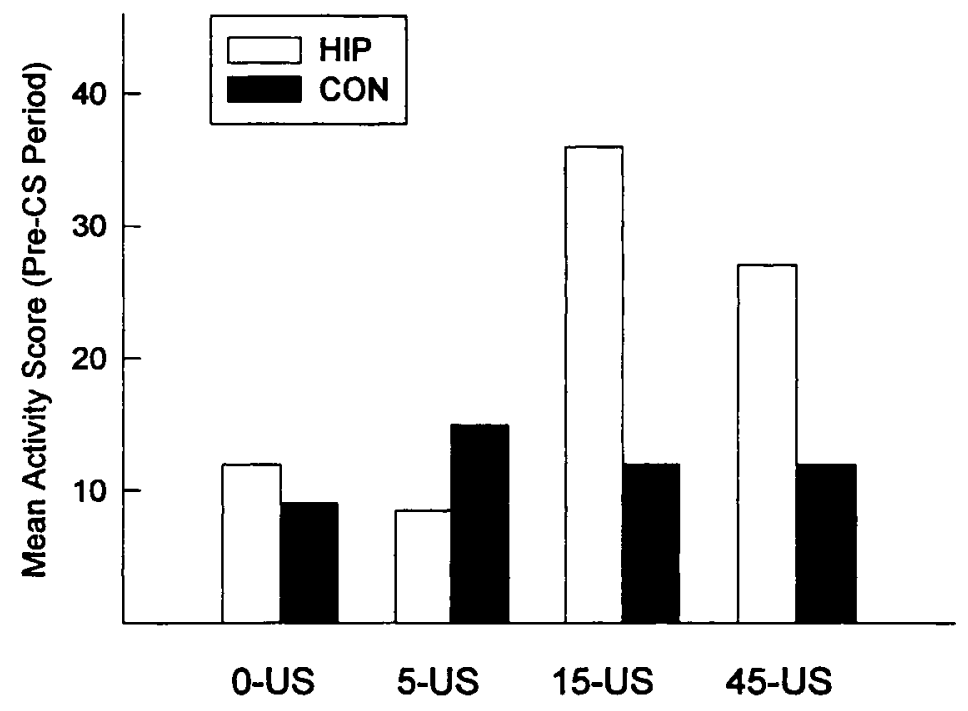

Figure 1. Mean activity scores (pre-CS period only) during the final block of acquisition in Experiment 1. Open bars represent data for animals with lesions of the hippocampus. Filled bars represent data for controls. 


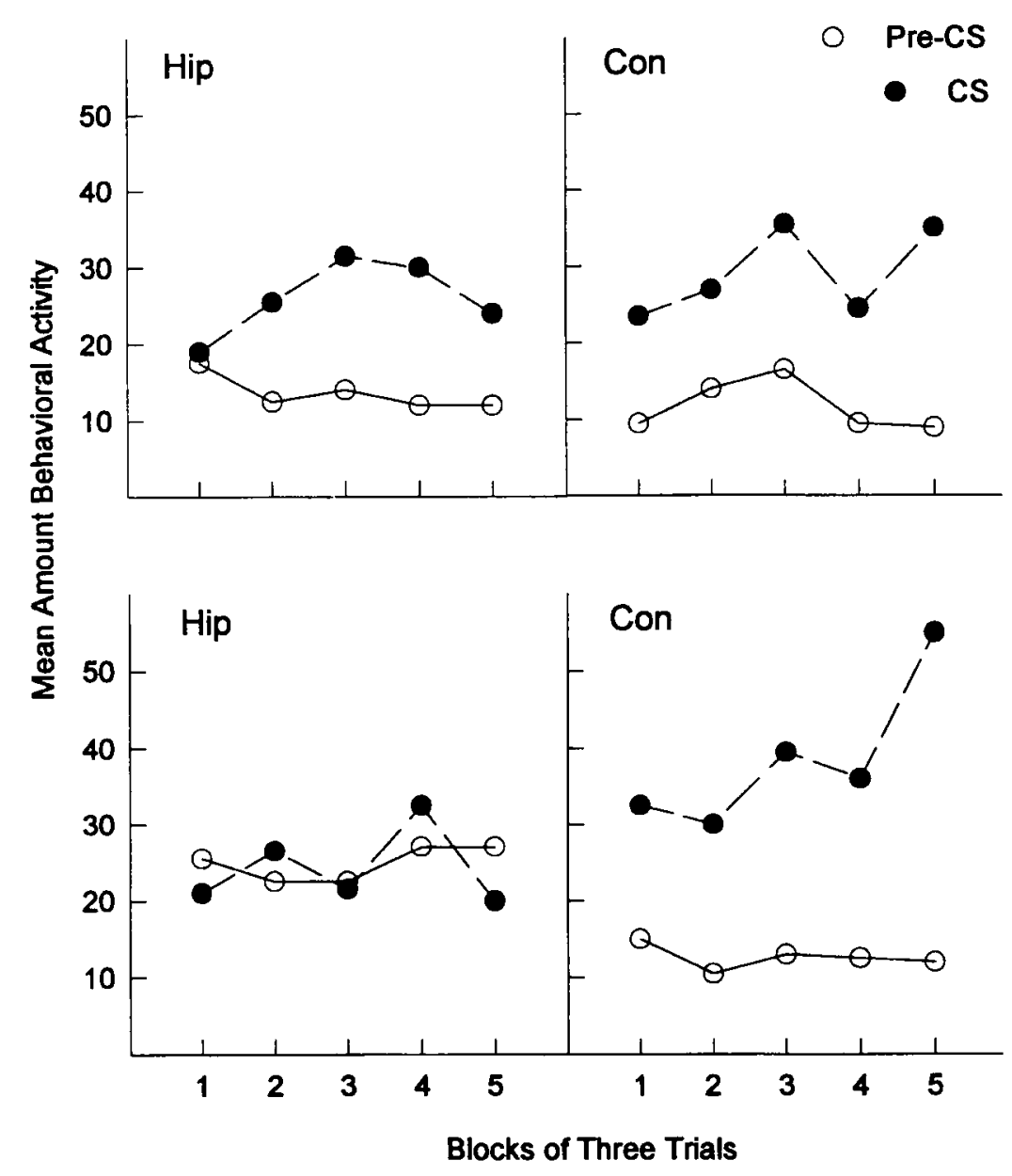

Figure 2. Mean activity scores in three trial blocks of pre-CS and CS during Experiment 1 . The top panels show data for hippocampally lesioned and control animals in Groups Few (0- and 5-US); the bottom panels show data for hippocampally lesioned and control animals in Groups Many (15- and 45-US). Open circles represent data for pre-CS periods. Closed circles represent CS data.

reliably different from one another, nor were Groups 15 and 45-US. Therefore, to increase statistical power, Groups 0 - and 5-US were combined for analyses and labeled Group Few. Groups 15- and 45-US were combined and labeled Group Many.

Figure 2 shows the mean activity score during the 15 training sessions in blocks of three trials. The top panels show pre-CS and CS activity scores for hippocampally lesioned and control animals in Group Few. The bottom panel shows activity for hippocampally lesioned and control animals in Group Many. Across sessions of training, control animals showed more behavioral activity during the CS than during the pre-CS period regardless of the number of unsignaled USs received. Further, behavioral activity during the CS period increased as a function of acquisition blocks. The amount of conditioned behavioral activity in lesioned animals, on the other hand, depended on the number of unsignaled US presentations. Lesioned animals exhibited the same pattern of data as that for controls when only zero or five unsignaled USs had been delivered in the training context. Lesioned animals in Group Many, on the other hand, exhibited as much behavioral activity during the pre-CS period as during the CS period, or more.

An ANOVA with group (Few vs. Many), lesion (hippocampal lesion vs. control), period (pre-CS vs. CS), and block (1-5) as factors revealed a main effect of group $[F(1,28)=4.52, p<.05]$ and a main effect of period $[F(1,28)=46.07, p<.01]$, as well as a lesion $\times$ period $[F(1,28)=11.59, p<.01]$ and a lesion $\times$ group $\times$ period $[F(1,28)=7.97, p<.01]$ interactions. Individual ANOVAs with lesion (hippocampal lesion vs. control), period (preCS vs. CS), and block (1-5) as factors were conducted on activity scores for each group, Few and Many. First, an ANOVA of data from Group Few revealed only a main effect of period $[F(1,14)=39.69, p<.05]$ and a margin- 


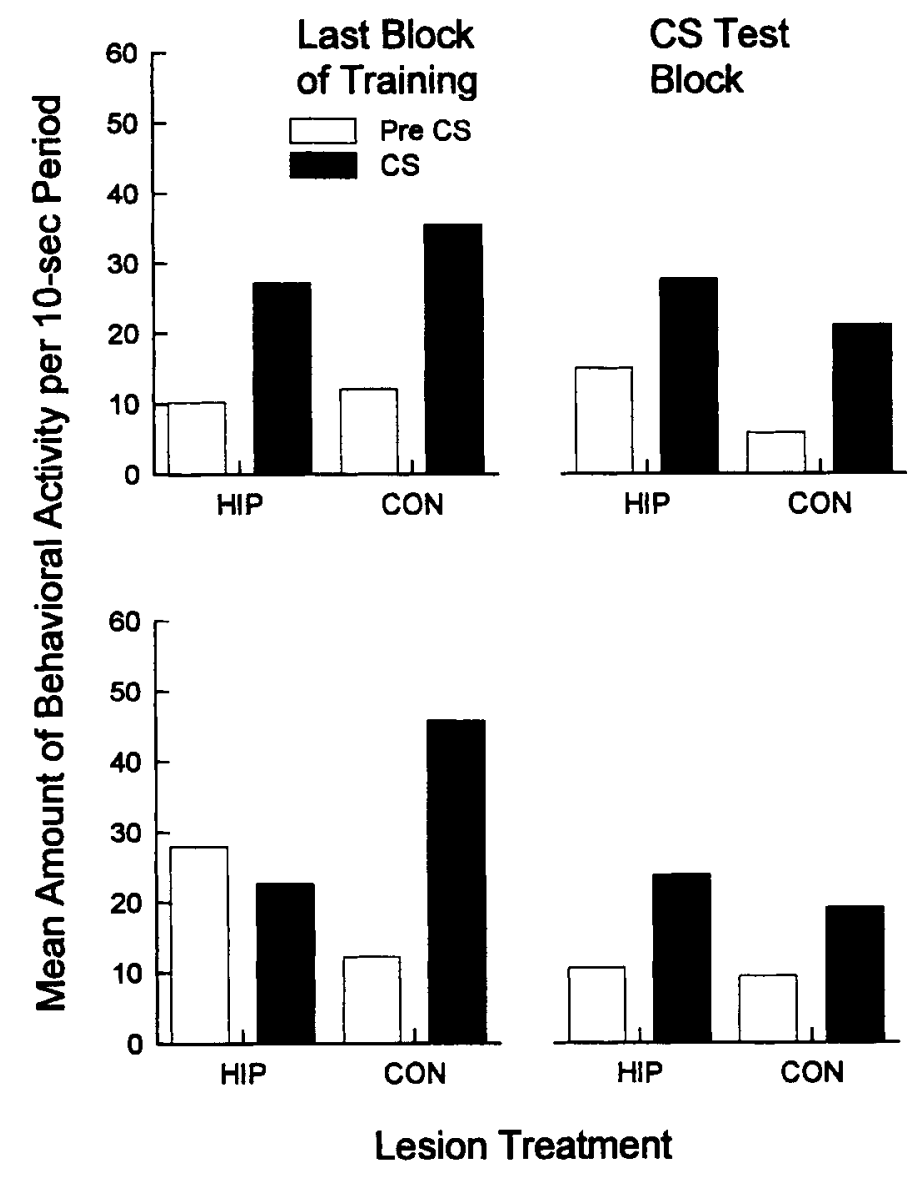

Figure 3. Mean activity scores (pre-CS and CS periods) during the final block of acquisition (left-hand panels) and tone-alone test trials (right-hand panels) in Experiment 1 . Top panels show data for Groups 0- and 5-US (Few). Bottom panels show data for Groups 15- and 45-US (Many). Open bars represent data for pre-CS periods. Filled bars show data for CS periods.

ally significant period $\times$ block interaction $[F(4,56)=$ $2.36, p<.06]$. No main effects or interactions involving lesion were found. An ANOVA with the same factors of data from Group Many, however, revealed a main effect of period $[F(1,14)=13.74, p<.01]$ as well as a lesion $X$ period interaction $[F(1,14)=15.5, p<.01]$. The bases for this interaction can be summarized in the analysis over the final block of training.

The left panels of Figure 3 show mean activity score during the final three-session block of training. The top panel shows data for animals in Group Few (0- and 5-US), while the bottom panel shows data for animals in Group Many (15- and 45-US). By this block, control animals exhibited more conditioned behavioral activity during the $\mathrm{CS}$ than during the pre-CS period, regardless of the number of unsignaled USs. Animals with selective IBO lesions of the hippocampus, however, showed a different pattern of behavioral activity. Hippocampally lesioned rats in Group Few, like controls, showed more conditioned activity during the CS than during the pre-CS period. Le- sioned animals in Group Many, however, did not show this pattern of behavioral activity.

First, a mixed $2 \times 2 \times 2$ ANOVA was conducted on behavioral activity from the final three-session block of acquisition, using lesion (hippocampal lesion vs. control), group (Few vs. Many), and period (pre-CS vs. CS) as factors. A reliable lesion $\times$ group $\times$ period interaction was found $[F(1,28)=7.75, p<.01]$ as well as a lesion $\times$ period interaction $[F(1,28)=14.54, p<.01]$ and a main effect of period $[F(1,28)=33.05, p<.01]$. Subsequent ANOVAs were then conducted on activity scores obtained during the final block of acquisition for each group (Few and Many). For Group Few, a reliable main effect of period $[F(1,14)=31.9, p<.01]$ was observed. However, neither a significant main effect of lesion nor a lesion $\times$ period interaction was obtained $\left(F_{\mathrm{s}}<1.5\right)$. For Group Many, a reliable main effect of period $[F(1,14)=$ $8.6, p<.01]$ as well as a significant lesion $\times$ period interaction $[F(1,14)=17.12, p<.01]$ was found. Subsequent post hoc tests revealed that control animals in 
Group Many showed reliably higher activity scores during the CS than during the pre-CS period. However, lesioned animals in Group Many did not show reliable differences between the two periods. Furthermore, post hoc testing revealed that lesioned animals showed reliably higher activity scores during the pre-CS period than did controls.

Finally, separate $2 \times 2$ mixed ANOVAs with period (pre-CS vs. CS) and Group (Few vs. Many) were performed on the activity scores obtained for each surgery condition (hippocampal lesion vs. control). For the control animals, the ANOVA yielded only a reliable main effect of period $[F(1,14)=34.21, p<.01]$. Neither a reliable main effect of group nor an interaction between period and group was observed, indicating that the control animals did not reliably differ in conditioned responding on the basis of membership in different US Groups. However, a significant period $\times$ group interaction was obtained for the lesioned animals $[F(1,14)=11.2, p<.01]$. Post hoc Newman-Keuls tests revealed that the hippocampally lesioned animals in Group Few showed more behavioral activity during the CS than did the lesioned animals in Group Many. Furthermore, the lesioned animals in Group Many showed significantly higher activity scores during the pre-CS period than did the lesioned animals in Group Few.

\section{Novel Context Test}

The right two panels of Figure 3 show mean activity score obtained during the three-session test block in the novel context. As in the final block of training, both hippocampally lesioned and control animals in Group Few exhibited greater amounts of behavioral activity during the $\mathrm{CS}$ than during the pre-CS period. Unlike in the final block of training however, the lesioned animals in Group Many did not differ from their control counterparts. Indeed, in the novel context, all animals, regardless of lesion group or number of unsignaled US deliveries, showed greater conditioned behavioral activity during the CS than during the pre-CS period.

A mixed $2 \times 2 \times 2$ ANOVA with lesion (hippocampal lesion vs. control), group (Few vs. Many), and period (pre-CS vs. CS) as factors evaluated the activity scores that were obtained during testing in the novel context. No reliable interactions involving lesion or group were found. However, a main effect of period $[F(1,28)=9.63$, $p<.01]$ and a main effect of lesion $[F(1,28)=4.32, p<$ $.05]$ were found. Subsequent Newman-Keuls post hoc tests revealed that all groups showed reliably more behavioral activity during the CS than during the pre-CS period.

\section{Anatomy}

The nature and extent of the resulting brain damage can be seen in Figure 4. These photomicrographs are cell-stained sections taken from four anterior-posterior levels through the hippocampus in a representative control and a representative IBO lesioned rat. Every animal that received an IBO lesion of the hippocampus had extensive loss of the pyramidal (CA 1-3) and dentate granule cells at all anterior-posterior levels. Any sparing of pyramidal cells was limited to a few CA1 cells at the dorsal level, and this was unilateral and present in only 2 animals. One rat had a small "island" of spared granule cells bilaterally at the most posterior extent of the hippocampus, and 2 rats had some minimal unilateral sparing. In every rat, any sparing was limited to a few cells. The other rats had complete removal of the hippocampus similar to that shown in Figure 4. A comparison of the behavioral data for the rats with some sparing and those with complete hippocampal lesions indicated no behavioral differences. It has been pointed out that even with minimal sparing similar to that described above, the hippocampus would probably be nonfunctional (David Amaral, personal communication).

As can be seen in Figure 4, lesioning the hippocampus with IBO results in extensive atrophy of the structure. The axons that normally pass through the alveus and fimbria can be seen forming a tight bundle of fibers along the dorsal and lateral edge of the thalamus. Results from a previous study indicated that these fibers of passage are still functional (see Jarrard, 1989). Specifically, rats that had the hippocampus removed with IBO 4 months earlier had HRP injected into the mamillary bodies of the hypothalamus, and the number and distribution of HRP-labeled cells in the subiculum was determined. Similar labeling of cells in the subiculum was found in rats that had the hippocampus removed earlier and in unoperated control rats. Thus, the extensive subicular projections that pass through the alveus and fimbria and terminate in the mamillary bodies are not interrupted when the hippocampus is removed with multiple injections of IBO.

\section{Discussion}

Rats with selective IBO lesions of the hippocampus showed greater amounts of activity during pre-CS periods than did controls only if they had been trained with relatively many ( 15 or 45 ) unsignaled US presentations. Removing the hippocampus did not elevate activity relative to controls when few ( 0 or 5 ) unsignaled presentations of the US occurred during training. In contrast, hippocampally lesioned rats did not show greater amounts of activity than did controls during the CS period regardless of the number of unsignaled USs that were presented during training. Furthermore, neither removal of the hippocampus nor number of unsignaled USs delivered during training had any significant effect on responding during either the pre-CS or the CS period when the rats were tested in a novel context than had not been subject to prior training with the CS or with unsignaled presentations of the US. The latter finding indicates that rats without a hippocampus did not differ from controls with respect to learning about the CS.

The findings of Experiment 1 argue against the notion that removing the hippocampus produces an increase in nonspecific or general behavioral activity as has previously 

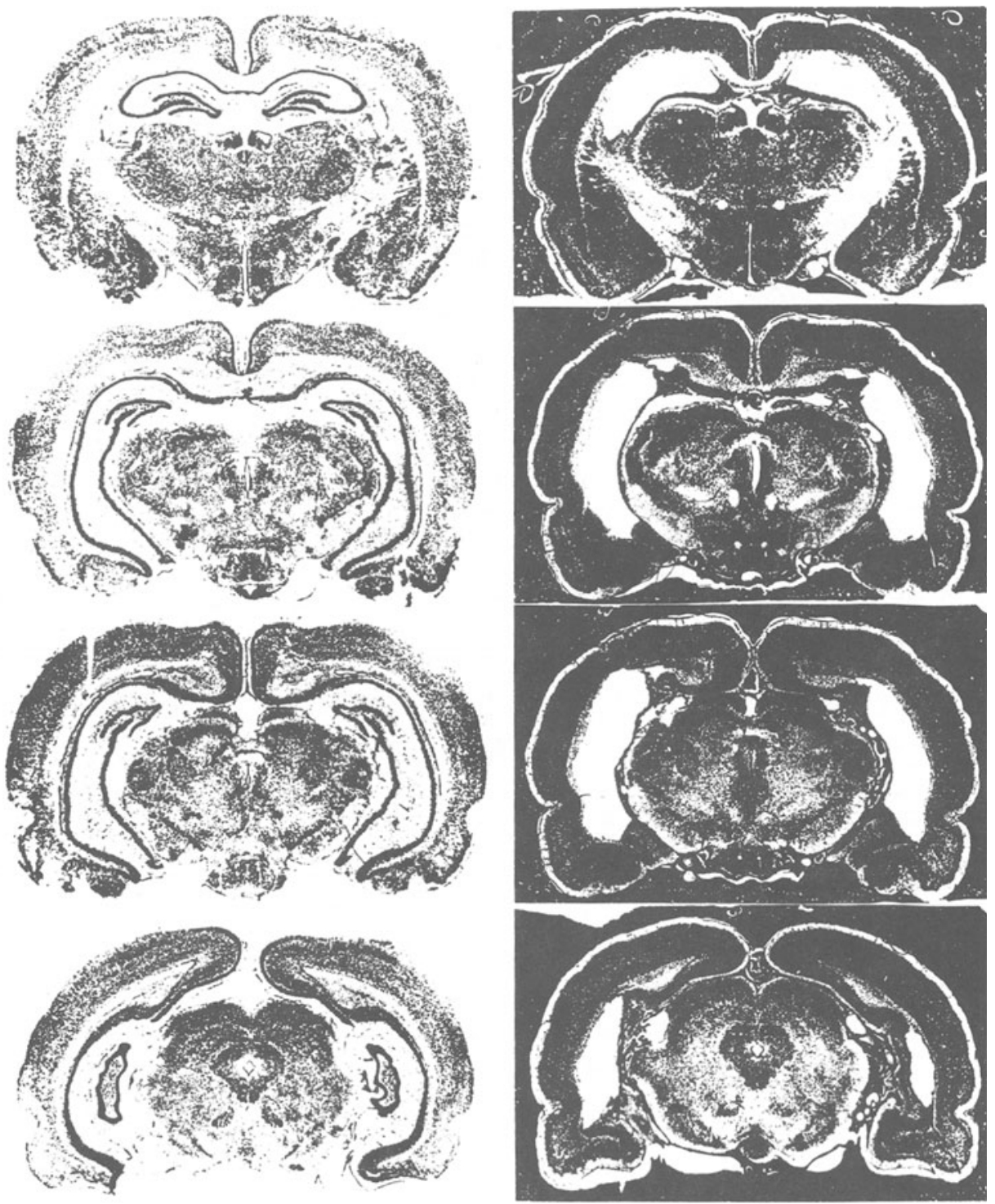

Figure 4. Coronal brain sections from a representative control (left) and a hippocampally lesioned rat (right) at four anterior-posterior levels. Note the cell fields that form the hippocampus (CA1-CA3 pyramidal cells, dentate gyrus) have been removed with minimal damage to extrahippocampal structures. With the loss of the hippocampus, axons that normally pass through the alveus and fimbria can be seen forming a tight bundle of fibers along the dorsal and lateral edge of the thalamus.

been suspected. Removing the hippocampus increased activity only in rats that had received relatively many unsignaled USs, and then only in the context where the unsignaled USs had been presented. Furthermore, it does not appear that removing the hippocampus increases excitatory conditioning in general. Rats without a hippocampus did not show more conditioned responding than did controls during presentations of the CS. These data seem most consistent with the hypothesis that removing the hippocampus enhanced the evocation of conditioned activity by cues in the training context. This effect of hippocampal damage appeared to be greater as a function of amount of context cue training with unsignaled presentations of the US. 


\section{EXPERIMENT 2}

The purpose of Experiment 2 was to investigate whether removing the hippocampus alters the learning or the performance of conditioned responses. If the hippocampus is critical for the formation of context-US associations but is not necessary for the expression of such learning in performance, then removing the hippocampus after learning has occurred should have little effect on conditioned responding. On the other hand, if the hippocampus is necessary for utilization of previously acquired context-US associations, then removing the hippocampus after learning has occurred would still be expected to influence the performance of conditioned responses.

In Experiment 2, we also examined the effects of removing the hippocampus on responding to a punctate CS. However, we assessed the effects of hippocampal damage during extinction rather than during acquisition of Pavlovian conditioned responding. Examining the effects of hippocampal lesions on extinction of a punctate CS may provide information about the mechanisms underlying the effects of removing the hippocampus on responding to conditioned contextual stimuli. Training with unsignaled presentations of the US can be seen as involving both the excitatory conditioning and the extinction of context cues (see, e.g., Rescorla \& Wagner, 1972). Although unsignaled presentations of the US promote excitatory conditioning of the context, the continued presence of the context during the time between US presentations should produce extinction of context cues. It may be the case that removing the hippocampus interferes with the extinction of conditioned responses, independently of whether those responses are evoked by contextual or punctate CSs. If so, rats without a hippocampus should show more responding than should controls during extinction of the punctate CS, just as they respond more than controls to context cues that have been subjected to both training and extinction.

In Experiment 2, Pavlovian training of a punctate CS was carried out using the same procedures as in Experiment 1 . In addition, the number of unsignaled presentations of the US was also varied during training, One half of the rats received 45 unsignaled USs and the other half received no unsignaled USs. After completing 15 sessions of training, half the rats in each group received IBO lesions of the hippocampus; the other half in each group remained as controls. Following recovery from surgery, conditioned responding during both the CS and pre-CS periods was assessed during several sessions of extinction training in which no USs, either signaled or unsignaled, were delivered.

\section{Method}

\section{Subjects}

As in Experiment 1, the subjects were 32 naive, male SpragueDawley albino rats obtained from Harlan Sprague-Dawley. The rats weighed approximately $275-300 \mathrm{~g}$ upon arrival in the laboratory. All other subject information was identical to that in Experiment 1 .
Table 2

Experiment 2 Design

\begin{tabular}{rrcc}
\hline Group & $N$ & CS-US Training & CS Testing \\
\hline 0-US & 16 & 0 Unsignaled USs per session & CS - \\
45-US & 16 & 3 Unsignaled USs per session & CS- \\
\hline
\end{tabular}

Note-One half of the rats in each group $(n=8)$ received ibotenate lesions of the hippocampus prior to CS Testing. Remaining rats were sham operated or unoperated controls. During training, all rats received one CS--US pairing per session. Training and test session length = $50 \mathrm{~min} . \mathrm{CS}=10-\mathrm{sec}$ tone. US $=$ two $45-\mathrm{mg}$ Noyes food pellets.

\section{Apparatus}

The apparatus in Experiment 2 was identical to that in Experiment 1 .

\section{Behavioral Procedures}

Training. Training began approximately 14 days after the rats arrived at the laboratory, and after the body weights of all rats were reduced to $85 \%$ of ad-lib feeding levels. As in Experiment 1 , all rats received 15 daily 50 -min training sessions. No events were programmed to occur during the first $20 \mathrm{~min}$ of each training session. During the final $30 \mathrm{~min}$ of each session, all rats received one $10-\mathrm{sec}$ tone CS immediately followed by a US (two 45-mg Noyes food pellets). Behavioral activity, measured as in Experiment 1, was assessed during each 10 -sec tone $\mathrm{CS}$ as well as during the $10 \mathrm{sec}$ immediately preceding tone CS presentation.

In addition to the single tone CS-US pairing per session, one half of the rats also received unsignaled US presentations. Table 2 shows the design for Experiment 2. Groups are labeled in Table 2 with respect to the total number of unsignaled USs that were delivered during training. Thus, Group 0 -US received 0 unsignaled USs during training, whereas Group 45-US received 3 unsignaled USs per session, for a total of 45 unsignaled US presentations during the 15 sessions of training. As in Experiment 1, unsignaled US presentation times were programmed at intervals of no less than $2 \mathrm{~min}$. Unsignaled USs were also programmed not to occur within 2 min of the training trials.

Extinction testing. After training but prior to extinction testing, one half of the rats in each group ( 0 - and 45-US) were randomly selected to receive IBO lesions of the hippocampus. Approximately 2 weeks after the rats had recovered from surgery, all rats were returned to the training context for extinction tests. All rats received 14 daily 50 -min extinction sessions in the training context. During these sessions, no events were programmed to occur during the first $20 \mathrm{~min}$. During the final $30 \mathrm{~min}$ of each session, all rats received one tone extinction trial, after which no US was delivered. No unsignaled USs were delivered during extinction testing.

\section{Surgical and Anatomical Procedures}

The procedures for IBO lesions of the hippocampus and for histological assessment of damage to the hippocampus and adjacent structures were the same as those described in Experiment 1.

\section{Results}

\section{Training (Presurgery)}

All rats exhibited more behavioral activity during the CS than during the pre-CS period by the end of training. Furthermore, the amount of conditioned responding during the CS and pre-CS periods did not depend on the number of unsignaled presentations of the US during training. Over the last three-trial block of training, the mean activity scores of the rats that received no unsignaled presentations were 42.87 during the CS and 16.78 during 


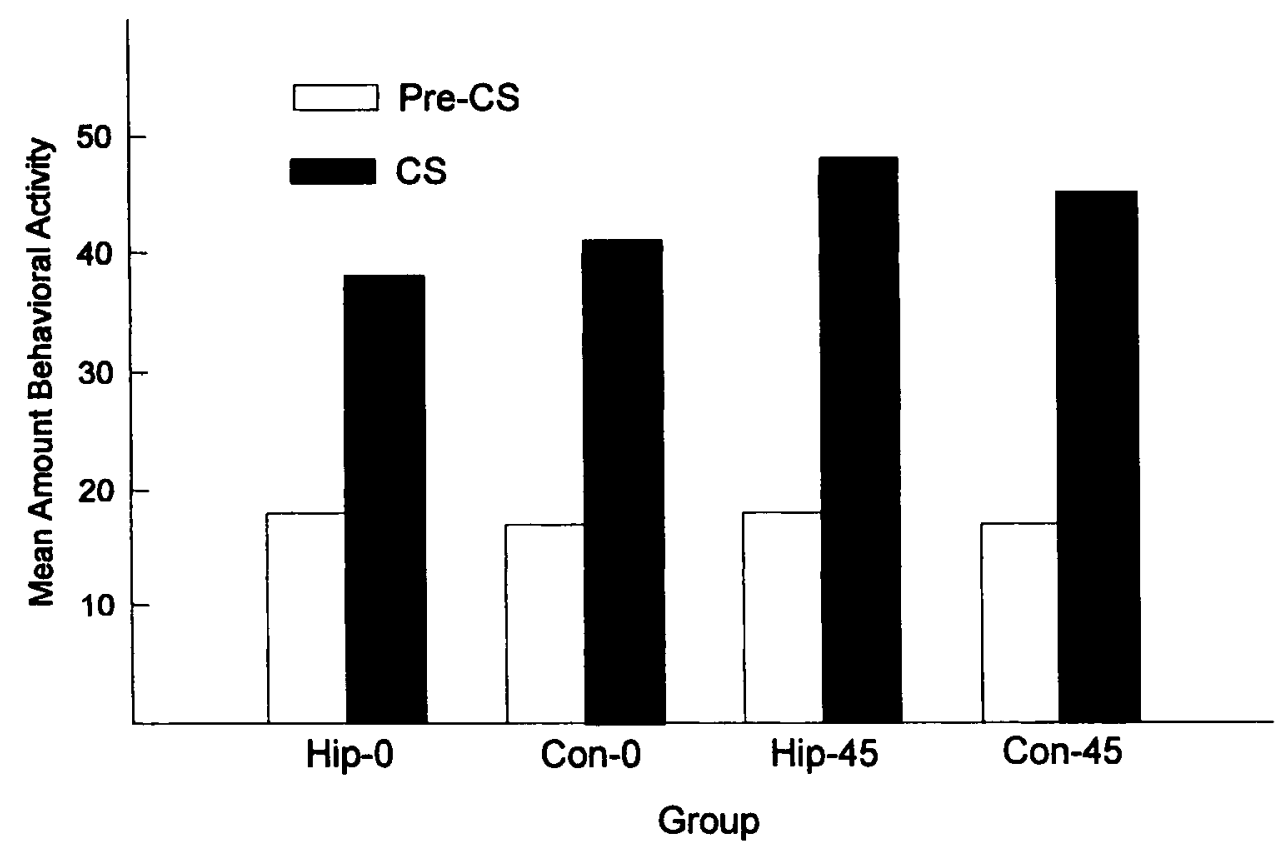

Figure 5. Mean activity scores (pre-CS and CS periods) during the first two-trial block of extinction testing during Experiment 2. Data are presented separately for both lesioned and control animals in US groups 0 and 45. Open bars show data for pre-CS period. Filled bars show data for CS period.

the pre-CS period. For rats that received 45 unsignaled presentations of the US, mean activity scores were 49.27 and 19.45 during the CS and pre-CS periods, respectively. An ANOVA with US group (0 vs. 45 unsignaled USs) and periods (CS vs. pre-CS) as factors yielded a highly significant main effect of period $[F(1,27)=109.92]$, but no significant interaction of period $\times$ US group $[F(1,27)<1]$. Thus, by the end of training, more activity was observed during the CS than during the pre-CS period, and this difference did not depend on the number of unsignaled presentations.

\section{Extinction Testing (Postsurgery)}

Figure 5 shows the mean activity scores obtained during the CS and pre-CS periods on the first two-trial block of extinction testing. The data are presented separately for rats with hippocampal lesions and controls as a function of the number of unsignaled presentations of the US ( 0 or 45 ) that occurred during training. As can be seen in the figure, all groups exhibited greater behavioral activity during the CS than during the pre-CS period. Of special interest is the finding that the responding of rats without a hippocampus did not differ from that of controls during either the pre-CS or the CS period and did not vary as a function of the number of unsignaled US presentations during training. Thus, removal of the hippocampus after training had been completed had little effect on retention of conditioned responding evoked by either punctate or contextual CSs.
A $2 \times 2 \times 2$ mixed ANOVA with lesion (hippocampal lesion vs. control), US group ( 0 vs. 45 ), and period (pre-CS vs. CS) as factors yielded a significant main effect of period $[F(1,25)=56.57, p<.01]$, confirming that the rats exhibited more behavioral activity during the CS than during the pre-CS period in the first block of extinction testing. No other main effects or interactions approached significance. Thus, there were no significant effects of hippocampal lesions or of unsignaled presentations of the US on the first block of extinction testing.

Figure 6 provides an assessment of conditioned responding for each treatment condition collapsed over the last two two-trial blocks of extinction testing. Extinction performance is depicted in terms of a CS minus pre-CS difference score. As Figure 6 shows, rats trained with 0 unsignaled USs tended to have higher activity difference scores (to respond more during the CS relative to the preCS period) than did rats trained with 45 unsignaled presentations of the US. This indicates that rats trained with 0 unsignaled presentations of the US were more resistant to the effects of extinction than were rats trained with 45 unsignaled presentations of the US. Figure 6 also reveals that, within each unsignaled US training condition, the superiority of responding during the CS relative to the pre-CS period was greater in rats without a hippocampus than in controls. Thus, greater resistance to extinction was exhibited by rats with hippocampal lesions than by controls. An ANOVA conducted over the last two blocks of extinction testing yielded significant main effects of le- 


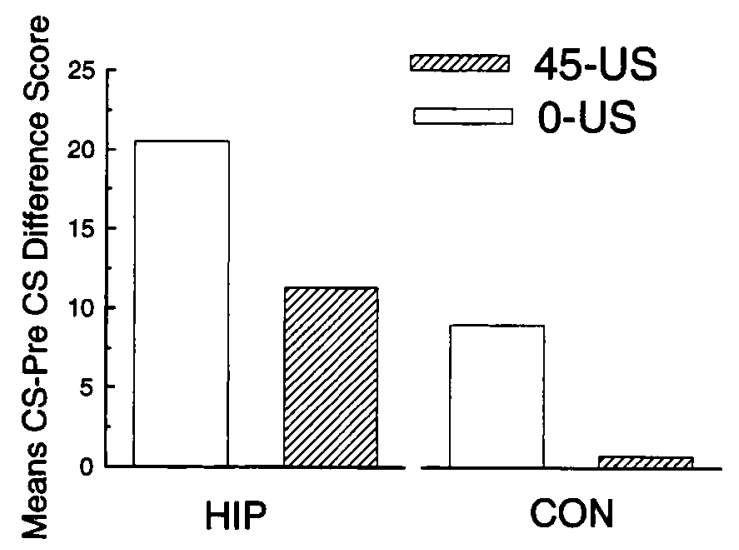

\section{Surgery}

Figure 6. Mean activity difference-scores (CS minus pre-CS periods) during the last two three-trial blocks of retention testing during Experiment 2. Open bars represent data for Groups 0-US. Hatched bars show data for Groups 45-US. The left panel shows data for animals with ibotenate lesions of the hippocampus. The right panel shows data for controls.

sion $[F(1,25)=7.85]$ and US group $[F(1,25)=12.51]$, whereas the interaction between these factors failed to attain significance $[F(1,25)<1]$.

Figure 7 shows mean activity score across all blocks of extinction testing for rats without a hippocampus and for controls, collapsed across US group. Extinction of responding to the punctate tone CS is evident; activity scores decrease for both groups across blocks of test sessions. However, extinction of responding to the tone appears to have been more rapid and complete in controls than in rats with hippocampal lesions.

These impressions were confirmed by a $2 \times 2 \times 7$ mixed ANOVA with lesion (hippocampal lesion vs. controls), period (pre-CS vs. CS), and block (1-7) as factors. This ANOVA yielded a significant lesion $\times$ block $\times$ period interaction $[F(6,150)=2.23, p<.05]$. Post hoc Newman-Keuls tests revealed no differences between lesioned rats and controls during the pre-CS periods on any block of testing. Although responding during CS periods did not differ between lesioned rats and controls during any of the first five blocks of extinction testing, rats without a hippocampus responded significantly more than controls during the CS on each of the last two blocks of testing. In addition, during the final two blocks of extinction testing, controls did not respond differently during the pre-CS and CS periods, whereas lesioned rats continued to show reliably greater activity scores during the CS than during the pre-CS periods.

\section{Anatomy}

The IBO-induced damage to the hippocampus was highly similar to that reported for Experiment 1. All IBO lesioned rats showed extensive loss of the pyramidal
(CA1-3) and dentate granule cells at all anterior-posterior levels, whereas damage to anatomically adjacent medial temporal structures was largely avoided. As in Experiment 1 , several rats had some minimal unilateral sparing of cells in CA1, and 2 rats had some unilateral sparing of dentate cells at the most posterior level. The performance of rats with this minimal sparing did not appear to differ from that found in rats that had the hippocampus removed completely.

\section{Discussion}

In Experiment 2, rats were trained with a punctate CS that signaled the delivery of a food pellet US. Half the rats also received additional unsignaled presentations of the US trained in the identical conditions. After training was completed, half the rats given unsignaled presentation of the US and half that did not receive the US unsignaled by the CS were given selective IBO lesions of the hippocampus. The behavioral activity of these rats during both the presence and the absence of the CS was then compared with that of the rats that had not received hippocampal lesions, during a test phase in which no USs, either signaled or unsignaled, were presented.

At the beginning of extinction testing, no differences in activity were observed between rats with the hippocampus removed and controls. Both lesioned and control rats showed significantly more activity during the CS than during the pre-CS period. Furthermore, both hippocampally lesioned rats and controls exhibited similar low levels of activity during the pre-CS periods of each trial and both groups showed similar and significantly higher levels of activity during the presentation of the CS. The amount of activity during the initial block of extinction testing did not depend on the number of unsignaled USs that were delivered during training nor did the effect of this factor depend on whether or not the hippocampus was removed.

However, significant effects of (1) training with unsignaled USs and (2) removing the hippocampus emerged by the end of the extinction test phase. Although the strength of conditioned responding to the CS decreased over the course of extinction testing for all rats, the magnitude of this decrease was less for rats that had been trained without unsignaled US presentations. By the end of extinction testing, rats that had not received unsignaled USs during training responded significantly more during the CS than during the pre-CS period, and more than did rats that received the US both in the presence and in the absence of the CS. This outcome indicates that presentation of unsignaled USs disrupted Pavlovian conditioning of the $\mathrm{CS}$ during training. More rapid extinction of conditioned responding during testing can be construed as evidence for weaker conditioning of the CS during initial training. This effect of presenting unsignaled USs during training was the same for rats without the hippocampus as it was for their nonlesioned controls. Thus, removing the hippocampus did not interfere with perfor- 


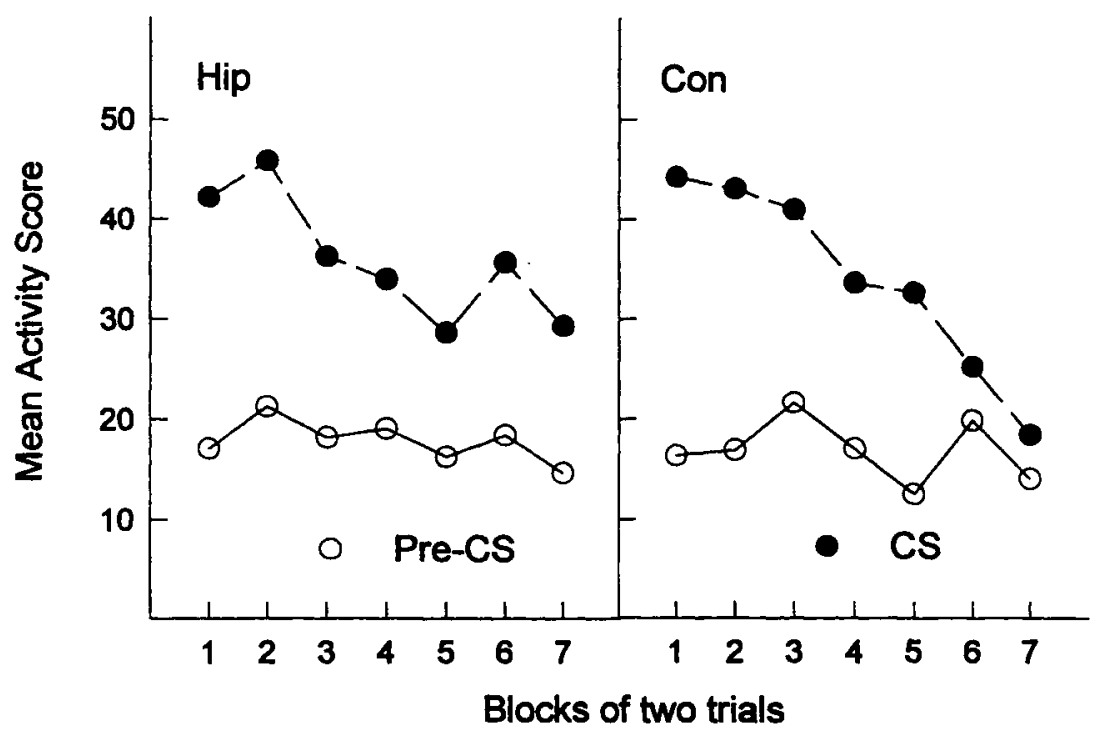

Figure 7. Mean activity scores (pre-CS and CS periods) during retention test extinction sessions in Experiment 2 collapsed across US groups. Data for animals with ibotenate lesions of the hippocampus are depicted in the left-hand panel. Data for control animals are depicted in the right-hand panel. Open circles show data for pre-CS periods. Closed circles show data for $\mathrm{CS}$ periods.

mance based on learning that occurred prior to when the hippocampus was removed.

It was also the case that responding during the CS period was reduced less by extinction in rats without a hippocampus than in controls. These groups did not differ at the beginning of extinction testing. However, by the end of testing, activity during the CS period and the elevation in responding during the CS relative to the pre-CS period were significantly greater in rats with the hippocampus removed than in controls. This effect of lesion was observed whether or not the rats had received unsignaled presentations of the US during training.

These results indicate that removing the hippocampus had little or no effect on performance based on learning that occurred prior to surgery. That is, the expression of prior learning about either the punctate CS or context cues (as assessed during pre-CS periods) did not require an intact hippocampus. Furthermore, the expression of the effects of prior cue competition between the punctate $\mathrm{CS}$ and conditioned contextual stimuli did not appear to depend on the structural integrity of the hippocampus. Rather, the results of Experiment 2 show only that removing the hippocampus influenced the learning that occurred during extinction testing.

\section{GENERAL DISCUSSION}

In the present experiments, the effects of unsignaled presentations of a food US on conditioned responding to a concurrently trained punctate CS were assessed in rats that had selective IBO lesions of the hippocampus. In
Experiment 1, rats without a hippocampus exhibited more behavioral activity than did controls during the $10-\mathrm{sec}$ period prior to the presentation of the CS. However, this effect of hippocampal damage was confined to rats that were trained with a relatively large number ( 15 or 45$)$ of unsignaled presentations of the US and was observed for these rats only in the context where training with the unsignaled USs was conducted. Hippocampal rats trained with either 0 or 5 unsignaled presentations did not show higher levels of pre-CS activity than did the controls. Furthermore, removing the hippocampus failed to augment the capacity of a punctate CS to evoke conditioned responding during training, regardless of the number of unsignaled presentations of the US. Thus, removing the hippocampus increased activity (1) only during the preCS period; (2) only for rats trained with relatively many unsignaled presentations of the US; and (3) only in the context where training with the unsignaled USs took place.

In Experiment 2, removing the hippocampus after the completion of excitatory training had little effect on subsequent pre-CS activity. However, responding to the punctate CS was more resistant to the suppressive effects of extinction in rats without a hippocampus than in controls. Furthermore, the results of extinction testing showed that both hippocampal and control rats showed similar sensitivity to the effects of unsignaled presentations of the US during training. That is, in lesioned rats in both US groups, responding to the CS was less resistant to extinction following training with 45 than with 0 unsignaled US presentations. This outcome is consistent with the 
hypothesis that unsignaled US presentations resulted in the formation of a weaker CS-US association in rats with and without a hippocampus.

It is difficult to attribute the findings of these studies to any nonspecific consequence of damage to the hippocampus. Hippocampal lesions produced increased activity relative to controls only in a context in which a relatively large number of unsignaled USs had been presented. Removing the hippocampus did not elevate activity if the context was subject to no or relatively few unsignaled presentations of the US. Furthermore, rats that received hippocampal lesions after the completion of training did not exhibit elevated pre-CS activity regardless of whether they were trained with many or few unsignaled deliveries of the US. Thus, only rats that were trained without a hippocampus exhibited elevated pre-CS activity and then only if they were trained with a relatively large number of unsignaled US deliveries.

On the other hand, the fact that selective lesions of the hippocampus elevated pre-CS activity only in rats that were trained with a relatively large number of unsignaled presentations of the US indicates that those lesions increased the capacity of the excitatory context cues to evoke conditioned activity. It may be that training with 0 or 5 unsignaled USs generated little or no excitatory conditioning of the context, whereas the presentation of 15 or 45 unsignaled USs exceeded the amount necessary for the formation of context-US associations. Damage to the hippocampus may have made it easier for excitatory context cues to evoke conditioned activity.

A number of theories of hippocampal function seem to propose that learning about contextual cues involves different processes than does learning about punctate stimuli. For example, some have suggested that different learning systems may be involved with the processing of contextual and punctate CSs (e.g., Myers \& Gluck, 1994; Rudy $\&$ Sutherland, 1994). These views have been encouraged by data indicating that removing the hippocampus has different effects on context learning relative to learning about discrete CSs (e.g., Kim \& Fanselow, 1992; Phillips \& LeDoux, 1992; Winocur et al., 1987; but see Good \& Honey, 1991). In contrast, in some prominent theories of Pavlovian conditioning, quite similar principles and concepts have often been applied to account for learning about both types of stimuli (e.g., Pearce \& Hall, 1980; Rescorla \& Wagner, 1972) Thus, from this perspective, hippocampal damage might be expected to have similar effects on learning about contextual and discrete CSs.

Although hippocampal damage appeared to have divergent effects on performance controlled by contextual and punctate CSs during both acquisition and extinction, it may still be possible to account for those effects in a unitary fashion within a basic Pavlovian conceptual framework. A straightforward approach would be to consider the possibility that removing the hippocampus produced some change in the capacity for US, cue (i.e., context and CS), or associative processing.
Some changes in the processing of the US, the conditioned cues, or the associative relation between the cues and the US can be characterized as incremental; others, as decremental (e.g., Han, Gallagher, \& Holland, 1995). For example, an increment in US processing might be based on an increase in the vividness or intensity of the memory of the US. Other things being equal, the stronger or more intense the US representation, the greater the strength of conditioned responding. However, an increment in US processing does not appear to account adequately for the present results. Given this approach, one would anticipate greater responding to context cues in Experiment 1 and more resistance to extinction to the CS in Experiment 2, but one would also have expected that hippocampal rats would show greater responding to the CS in Experiment 1. That result was not obtained. Likewise, the idea that $C S$ and context cue processing was incremented in the present studies as a consequence of removing the hippocampus also encounters difficulty. Increased attention might account for enhanced extinction responding to the CS observed in Experiment 2, but it would not explain why rats with the hippocampus removed did not respond more than controls to the CS in Experiment 1. Finally, complications also face the notion that removing the hippocampus produces an increment in associative processing. Although in Experiment 1 the context may have been more strongly associated with the US in hippocampally lesioned rats than in controls, evidence for a difference in the strength of the CS-US association was not obtained. Moreover, differences in the associative processing of the excitatory CS-US relation could not account for the effects of removing the hippocampus on extinction of responding to the CS in Experiment 2.

In contrast to these incremental changes, hippocampal damage might influence performance by disrupting decrements that normally occur in the processing of the USs, cues, or their associations. In Experiment 1, both context cues and the punctate CS underwent excitatory conditioning, as a consequence of being present when USs were delivered. However, context cues also underwent extinction as a consequence of being present during the intervals between US presentations. Like these context cues, the punctate CS in Experiment 2 also received both excitatory training and extinction. Although removing the hippocampus appeared to have little effect on incremental processing induced by excitatory training, it may have impaired the decremental processing that resulted from extinction.

For example, one consequence of withholding the US during extinction could be the deterioration of the memory of the US. Conditioned responding to trained and extinguished context cues and punctate CSs would be elevated to the extent that hippocampal damage reduced or prevented this deterioration. However, impaired decremental US processing will not provide a complete account of the present results without explaining why the CS in 
Experiment 1 failed to evoke more responding in hippocampal than in control rats. If elevated pre-CS activity was based on reduced deterioration of the US memory, then responding to the $\mathrm{CS}$, which was also based on that US representation, should also have been elevated for lesioned rats. Thus, the data do not seem to support the notion that lesions of the hippocampus affected decremental US processing.

Another possibility is that IBO lesions of the hippocampus impair decremental cue processing. This hypothesis derives support from the finding, obtained elsewhere (Han et al., 1995), that hippocampal lesions reduce the loss of CS associability or attention that normally occurs as a consequence of nonreinforced preexposure to that CS (i.e., latent inhibition). However, the expected impact of this effect on responding during extinction is difficult to ascertain. For example, in Experiment 2, if rats with hippocampal lesions attended more to the CS than did controls, this might make it easier for the hippocampally lesioned rats to learn that the CS was followed by a new consequence (i.e., no US) and would thereby decrease rather than increase resistance to extinction. That is, impaired latent inhibition might yield faster extinction of conditioned responding.

Within the Pavlovian framework, the idea that seems to make the most sense of the present findings is that removing the hippocampus disrupts decremental associative processing. Several theorists proposed that one effect of extinction is the development of inhibitory associations between the CS and the US (e.g., Bouton, 1994) or between the CS and the memory of no US (e.g., Konorski, 1967). These inhibitory associations can coexist with the excitatory CS-US associations and thus reduce the capacity of the CS to evoke a conditioned response. If rats without a hippocampus were impaired in learning these inhibitory associations, they would tend to respond more than controls during CS extinction, as was found in Experiment 2. Lesioned rats would also be less able to form inhibitory context-US (or context-no US) associations. Amount of activity for hippocampally lesioned rats would then be determined largely by the strength of the excitatory context-US association. Because context cues for rats trained with 0 or 5 unsignaled USs would possess little excitation, they would evoke little conditioned responding even in the absence of inhibition. Rats trained with 15 and 45 unsignaled USs would have excitatory context-US associations that were of similar strength both with and without a hippocampus. However, the hippocampally lesioned rats would show greater preCS activity than would controls, because they would have weaker inhibitory associations involving context cues.

One corollary of this analysis is that previous reports of increased general activity in hippocampally lesioned animals are due to the existence of context-US associations. These associations are certainly developed during some explicit, appetitive training procedures, but might also arise through experimental events as seemingly innocuous as magazine training. Indeed, Dickinson and his colleagues have reported that such US (or outcome) exposure may be the basis for some food-deprivation manipulation phenomena in intact animals undergoing instrumental conditioning (Dickinson \& Dawson, 1988; see Dickinson \& Balleine, 1994, for full explanation). Therefore, it is not surprising that the present results appear to contradict, somewhat, previous reports of increased generalized activity, because in the present experiment we attempted to explicitly manipulate the presence or absence of such US presentations.

This analysis seems able to account for the present findings on the basis of the idea that removing the hippocampus disrupts the decremental associative processing involved with the formation of inhibitory associations between CSs and USs and context cues and USs, respectively. This approach may provide a viable alternative interpretation of the effects, or the lack thereof, of hippocampal damage in other simple conditioning situations. However, extending this view to other results will be complicated by two problems. First, this view will be applicable only to situations in which inhibitory learning occurs. Failure to respond, or low levels of responding, to a CS may indicate weak excitatory conditioning rather than inhibitory conditioning. In this situation, no effect of hippocampal damage is expected. Second, recent evidence suggests that that there may be more than one type of conditioned or learned inhibition, some of which are antagonistic to conditioned excitation, and some of which are not (for a review, see Williams, Overmier, \& LoLordo, 1992). Therefore, although the inhibitory learning produced by training and extinction may depend on an intact hippocampus, other forms of conditioned inhibition may not (see Solomon, 1977).

The present discussion has been limited to the role of the hippocampus in appetitive conditioning situations. Previous studies, however, have shown that removal of the hippocampus impairs conditioned responding to contextual stimuli that are present when shock is delivered (e.g., Kim \& Fanselow, 1992; Philips \& LeDoux, 1992). Contextual stimuli in aversive situations, however, should undergo both excitatory and inhibitory conditioning. In view of these findings, it is interesting that some prominent theories of animal learning propose that appetitive and aversive stimuli are functional opposites. That is, appetitive excitation is assumed to weaken aversive excitation, whereas appetitive inhibition is assumed to weaken aversive inhibition (e.g., Dickinson \& Dearing, 1979; Konorski, 1967). These assumptions would provide a unitary account of how removal of the hippocampus appears to produce impaired acquisition of inhibition in appetitive situations and enhances inhibition when conditioning is based on an aversive US. This hypothesis awaits further experimentation.

Finally, we have confined the present analysis to the role of the hippocampus in simple conditioning situationssituations in which the utilization of spatial information is minimized. The involvement of hippocampal-dependent inhibitory learning in complex tasks centering on spatial learning (see, e.g., Jarrard, 1993; Morris, Schenk, Tweedie, 
\& Jarrard, 1990; O’Keefe \& Nadel, 1978), in complex nonspatial tasks (e.g., Davidson \& Jarrard, 1989; Davidson, McKernan, \& Jarrard, 1991; Jarrard \& Davidson, 1995 ), and in problems that seem to involve learning about both spatial and nonspatial cues (e.g., Winocur et al., 1987; Selden et al., 1991) will not be discussed here, since little is known about how inhibitory learning develops within these more complex situations. Given our present findings, and the results of other hippocampal studies including those involving spatial learning, it would seem that one is forced to consider the possibility that the hippocampus plays an important role in several different behavioral processes (see Jarrard, 1973, for an early discussion of this point). Certainly, an important implication of the present results is that investigators should use caution in interpreting the results of hippocampal lesion studies when differences in conditioned inhibitory processes could affect the outcome of the research.

\section{REFERENCES}

Bouton, M. E. (1994). Context, ambiguity and classical conditioning. Current Directions in Psychological Sciences, 3, 49-53.

DavidSON, T. L., \& JaRRaRD, L. E. (1989). Retention of concurrent conditional discriminations in rats with ibotenate lesions of hippocampus. Psychobiology, 17, 49-60.

Davidson, T. L., McKernan, M. G., \& Jarrard, L. E. (1991). Hippocampal lesions do not impair negative patterning: A challenge to configural association theory. Behavioral Neuroscience, 107, 227-234.

Dickinson, A., \& Balleine, B. (1994). Motivational control of goaldirected action. Animal Learning \& Behavior, 22, 1-18.

Dickinson, A., \& Dawson, G. R. (1988). Motivational control of instrumental performance: The role of prior experience of the reinforcer. Quarterly Journal of Experimental Psychology, 40B, 113-134.

Dickinson, A., \& Dearing, M. F. (1979). Appetitive-aversive interactions and inhibitory processes. In A. Dickinson \& R. A. Boakes (Eds.), Mechanisms of learning and motivation: A memorial volume to Jerzy Konorski (pp. 203-231). Hillsdale, NJ: Erlbaum.

Eichenbaum, H., Otto, T., \& Cohen, N. J. (1992). The hippocampus: What does it do? Behavioral \& Neural Biology, 57, 2-36.

Gibbon, J., \& Balsam, P. D. (1980). Spreading association in time. In C. M. Locurto, H. S. Terrace, \& J. Gibbon (Eds.), Autoshaping and conditioning theory (pp. 219-253). New York: Academic Press.

Good, M., \& HonEY, R. C. (1991). Conditioning and contextual retrieval in hippocampal rats. Behavioral Neuroscience, 105, 499-509.

HaN, J.-S., Gallagher, M., \& Holland, P. (1995). Hippocampal lesions disrupt decrements but not increments in conditioned stimulus processing. Journal of Neuroscience, 15, 7323-7329.

HONEY, R. C., \& GoOD, M. (1993). Selective hippocampal lesions abolish the contextual specificity of latent inhibition and conditioning. Behavioral Neuroscience, 107, 23-33.

JARRARD, L. E. (1973). The hippocampus and motivation. Psychological Bulletin, 79, 1-12.

JARRARD, L. E. (1989). On the use of ibotenic acid to lesion selectively different components of the hippocampal formation. Journal of Neuroscience Methods, 29, 251-259.

JARRARD, L. E. (1993). On the role of the hippocampus in learning and memory in the rat. Behavioral \& Neural Biology, 80, 9-26.
JARRARD, L. E., \& Davidson, T. L. (1995). The hippocampus and nonspatial discrimination: Is learning still "not possible"? In N. E. Spear, L. S. Parker, \& M. L. Woodruff (Eds.), Neurobehavioral plasticity (pp. 15-32). Hillsdale, NJ: Erlbaum.

KIM, J. J., \& FANSELow, M. S. (1992). Modality-specific retrograde amnesia of fear. Science, 256, 675-677.

KONORSKI, J. (1967). Integrative activity of the brain. Chicago: University of Chicago Press.

MilleR, R. R., \& MATzEL, L. D. (1988). The comparator hypothesis: A response rule for the expression of associations. In G. H. Bower (Ed.), The psychology of learning and motivation (Vol. 22, pp. 51-92). San Diego, CA: Academic Press.

MorRIS, R. G. M., Schenk, F., Tweedie, F., \& JarRard, L. E. (1990). Ibotenate lesions of hippocampus and/or subiculum: Dissociating components of allocentric spatial learning. European Journal of Neuroscience, 2, 1016-1028

Myers, C. E., \& Gluck, M. A. (1994). Context, conditioning, and hippocampal rerepresentation in animal learning. Behavioral Neuroscience, 108, 835-847.

O'KEEFE, J., \& NADEL, L. (1978). The hippocampus as a cognitive map. Oxford: Oxford University Press.

Pearce, J. M., \& Hall, G. (1980). A model for Pavlovian learning: Variations in the effectiveness of conditioned but not of unconditioned stimuli. Psychological Review, 87, 532-552.

Philips, R. G., \& LeDoux, J. E. (1992). Differential contributions of amygdala and hippocampus to cues and contextual fear conditioning. Behavioral Neuroscience, 106, 274-285.

RaWLiNs, J. N. P. (1985). Associations across time: The hippocampus as a temporary memory store. Behavioral \& Brain Sciences, 8, 479-496.

Rescorla, R. A. (1968). Probability or shock in the presence and absence of CS in fear conditioning. Journal of Comparative $\&$ Physiological Psychology, 66, 1-5.

Rescorla, R. A., \& WAGNER, A. R. (1972). A theory of Pavlovian conditioning: Variation in the effectiveness of reinforcement and nonreinforcement. In A. H. Black \& W. F. Prokasy (Eds.), Classical conditioning II: Current research and theory (pp. 64-99). New York: Appelton-Century-Crofts.

Rudy, J. W., \& SuTherland, R. J. (1994). The memory-coherence problem, configural associations, and the hippocampal system. In D. L. Schacter \& E. Tulving (Eds.), Memory systems 1994 (pp. 119 146). Cambridge, MA: MIT Press, Bradford Books

SELDON, N. R. W., EveritT, B. J., JarRaRd, L. E., \& Robins, T. W. (1991). Complementary roles for the amygdala and hippocampus in aversive conditioning to explicit and contextual cues. Neuroscience, 42, 335-350.

Solomon, P. R. (1977). Role of hippocampus in blocking and conditioned inhibition of rabbit's nictitating membrane response. Journal of Comparative \& Physiological Psychology, 91, 407-417.

Williams, D. A., Overmier, J. B., \& LoLordo, V. M. (1992). A reevaluation of Rescorla's early dictums about Pavlovian conditioned inhibition. Psychological Bulletin, 111, 275-290.

Wilson, A., Brooks, D. C., \& Bouton, M. E. (1995). The role of the rat hippocampal system in several effects of context in extinction. Behavioral Neuroscience, 109, 828-836.

Winocur, G., RaWlins, J. N. P., \& Gray, J. A. (1987). The hippocampus and conditioning to contextual cues. Behavioral Neuroscience, 101, 617-625.

(Manuscript received November 18, 1997; revision accepted for publication March 31, 1998.) 